\title{
Exploratory study on relative dose intensity and reasons for dose reduction of adjuvant CAPOX therapy in elderly patients with colorectal cancer
}

\author{
Ayumi Tsuchiya, ${ }^{1, *}$, Chiaki Ogawa', Naoki Kondo', Yasushi Kojima², Yasuhide Yamada ${ }^{3}$, Hiroyuki Terakado' \\ ${ }^{1}$ Pharmaceutical Department, Center Hospital of the National Center for Global Health and Medicine, Tokyo, Japan; \\ ${ }^{2}$ Gastroenterology, Center Hospital of the National Center for Global Health and Medicine, Tokyo, Japan; \\ ${ }^{3}$ Comprehensive Cancer Center, Center Hospital of the National Center for Global Health and Medicine, Tokyo, Japan.
}

\begin{abstract}
Capecitabine plus oxaliplatin, CAPOX, therapy is one of the standardized options for adjuvant chemotherapy for colorectal cancer, but the efficacy and the safety of CAPOX in elderly patients are unclear. In this study, we investigated the relative dose intensity (RDI) and reasons for dose reduction in patients over the age of 70 (elderly group) ( $n=12)$ and those under the age of 70 (non-elderly group) $(n=24)$ receiving adjuvant CAPOX therapy for colorectal cancer. The median RDIs were $71.1 \%$ in the elderly group and $67.9 \%$ in the non-elderly group for oxaliplatin ( $p=0.416)$, and $81.6 \%$ and $86.4 \%$ for capecitabine $(p=0.166)$, respectively. The rate of peripheral neuropathy which was the reason for dose reduction of oxaliplatin was approximately 4.5 -fold higher in the nonelderly group than in the elderly group. In addition, hematologic toxicity was the most common reason for dose reduction at $50.0 \%$ in the elderly group. The results of this study suggested that a similar therapeutic intensity can be maintained in elderly patients relative to non-elderly patients by appropriate dose reduction and discontinuation of drug treatments. Elderly patients are more susceptible to hematologic toxicity than to peripheral neuropathy.
\end{abstract}

Keywords: oxaliplatin, over the age of 70, therapeutic intensity, peripheral neuropathy, hematologic toxicity

\section{Introduction}

Cancer has been the leading cause of death in Japan since 1981. According to the latest cancer statistics from the Center for Cancer Control and Information Services, National Cancer Center, colorectal cancer was the most common cancer in 2017, and its ageadjusted incidence rate in people over the age of 70 was $59 \%$ in 2015 , accounting for more than half of the total incidence rate (1).

Surgery and adjuvant chemotherapy are regarded as the standard treatment for resectable colorectal cancer. CAPOX therapy, a combination of capecitabine and oxaliplatin, has been shown to be superior to fluorouracil $(\mathrm{FU})+$ levofolinate calcium ( $(\mathrm{-LV})$ therapy in terms of disease-free survival (DFS) (2). It has become one of the standardized options for adjuvant chemotherapy for colorectal cancer in Japan.

Adjuvant chemotherapy with an FU-based regimen has been shown to be effective regardless of age; however, the results of clinical trials evaluating the efficacy of the combined use of oxaliplatin are still controversial (3-5), and consistent results have not been obtained (6). Furthermore, peripheral neuropathy arises in a dose-dependent manner when oxaliplatin is used in combination $(7,8)$. There are only a few reports of adverse events in patients over the age of 70 that have been investigated to date, and they are often excluded from clinical trials because of worse performance status or complications. The Clinical Practice Guidelines of Cancer Drug Therapies for the Elderly (9) stipulate that "adjuvant chemotherapy may be considered for elderly patients with good performance status and organ function and no serious complications", but the efficacy and the safety of the CAPOX therapy in patients over the age of 70 have not been clarified.

In this study, we investigated the relative dose intensity (RDI) and reasons for dose reduction in patients over the age of 70 and those under the age of 70 receiving adjuvant CAPOX therapy for colorectal cancer.

\section{Patients and Methods}

\section{Patients}

Patients over the age of 18 who started CAPOX therapy as adjuvant chemotherapy for colorectal cancer at the Center Hospital of the National Center for Global 
Health and Medicine (NCGM) between January 2018 and August 2019 were included. Surgery was performed on all patients within 8 weeks. Of these patients, those who met the criteria of white blood cells $\geq 3,000 / \mathrm{mm}^{3}$, neutrophils $\geq 1,500 / \mathrm{mm}^{3}$, platelets $\geq$ $75,000 / \mathrm{mm}^{3}$, hemoglobin $\geq 8.0 \mathrm{mg} / \mathrm{dL}$, total bilirubin $\leq 1.5 \mathrm{mg} / \mathrm{dL}$, and serum creatinine $\leq 1.5 \mathrm{mg} / \mathrm{dL}$ at the start of treatment were included. Other eligibility criteria included an Eastern Cooperative Oncology Group Performance Status of 0/1. Patients with known peripheral neuropathy were excluded.

\section{Methods}

CAPOX therapy consisted of 8 courses of treatment with each course as a 21-day cycle, administering $130 \mathrm{mg} / \mathrm{m}^{2}$ oxaliplatin on day 1 and $2,000 \mathrm{mg} / \mathrm{m}^{2}$ capecitabine per day from after dinner on day 1 to after breakfast on day 15 .

A retrospective survey was conducted based on electronic medical records. The severity of adverse events was assessed according to the Common Terminology Criteria for Adverse Events, version 5.0 (CTCAE ver.5.0). The RDI was calculated as (Actual total dose/Actual total administration period)/(Planned total dose/Planned total administration period) $\times 100$ (\%). Based on the Guidelines for the Treatment of Colorectal Cancer, "elderly" was defined as over the age of 70. Patients over the age of 70 (elderly group) was compared with those under the age of 70 (nonelderly group).

Baseline characteristics of patients, course of treatment, RDI (\%), reasons for dose reduction and discontinuation of oxaliplatin, and reasons for dose reduction of capecitabine were investigated.

\section{Statistical analysis}

The Mann-Whitney $U$ test was used to compare quantitative data, and the chi-square test or the Fisher's exact test was used to compare qualitative data. In all cases, $p<0.05$ was considered a statistically significant difference. IBM SPSS ${ }^{\circledR}$ Statistics ver.24 was used for all statistical analyses.

\section{Ethical conduct of the study}

This study was conducted with the approval of the Ethical Review Committee of NCGM (Approval number: NCGM-G-003546-01), in compliance with the "Ethical Guidelines for Medical and Health Research Involving Human Subjects" and paying the utmost attention to the protection of personal information.

\section{Results and Discussion}

\section{Baseline characteristics of the study patients}

The baseline characteristics of the 36 patients are shown in Table 1 . The median age of the 12 elderly patients and the 24 non-elderly patients was 74.0 and 52.0 , respectively. Laboratory data prior to the start of treatment indicated that hemoglobin and albumin were significantly lower in the elderly than in the non-elderly group ( $p=0.026$ and 0.022 , respectively).

\section{Course of treatment}

The courses of treatment for the 36 patients are shown in Figure 1. In total, $69.4 \%$ of the patients (25/36) completed the 8 courses. The rate of treatment completion in the elderly group $(n=12)$ and the nonelderly group $(n=24)$ was $58.3 \%(7 / 12)$ and $75.0 \%$ (18/24), respectively. The reasons for failure to complete treatment in the elderly-vs-non-elderly groups were recurrence in 1 and 4 patients; concomitant other diseases or progression of other diseases such as lung cancer in 4 and 0 patients; financial considerations in 0 and 1 patient; and a personal decision in 0 and 1 patient, respectively.

\section{Relative dose intensity (RDI) of CAPOX}

The median RDIs in the elderly and non-elderly patients who completed the 8 courses are shown in Figure 2. The RDIs of oxaliplatin were $71.1 \%$ and $67.9 \%$, respectively (not significant, $p=0.416$ ) and the RDIs of capecitabine were $81.6 \%$ and $86.4 \%$, respectively (also not significant, $p=0.166$ ).

\section{Reasons for dose reduction or discontinuation of oxaliplatin and capecitabine}

The reasons for dose reduction and discontinuation of oxaliplatin are shown in Table 2 and the reasons for dose reduction of capecitabine are shown in Table 3 . The rate of peripheral neuropathy which was the reason for dose reduction of oxaliplatin was $8.3 \%(1 / 12)$ in the elderly group but approximately 4.5 -fold higher in the nonelderly group $(37.5 \%$; 9/24). All patients discontinuing oxaliplatin due to peripheral neuropathy were in the non-elderly group, accounting for 29.2\% (7/24), with a median number of 6 oxaliplatin courses. In addition, in the elderly group, the most common reason for dose reduction of oxaliplatin was hematologic toxicity in $50.0 \%$ of the patients $(6 / 12)$.

\section{Discussion}

The actual condition of treatment in patients over the age of 70 who were treated with adjuvant CAPOX therapy for colorectal cancer at NCGM is reported here. The rate of treatment completion was lower in the elderly group than in the non-elderly group, but not all reasons for failure to complete treatment were due to adverse events. In addition, there was no difference in 
Table 1. Baseline characteristics of patients

\begin{tabular}{|c|c|c|c|}
\hline Characteristic & Elderly $(n=12)$ & Non-Elderly $(n=24)$ & $P$ value \\
\hline \multicolumn{4}{|l|}{ Age, years } \\
\hline Median (Range) & $74.0(70.0-77.0)$ & $52.0(27.0-67.0)$ & \\
\hline Sex & & & 0.451 \\
\hline Male & $7(58.3 \%)$ & $17(70.8 \%)$ & \\
\hline Female & $5(41.7 \%)$ & $7(29.2 \%)$ & \\
\hline \multicolumn{4}{|l|}{ Body surface area, $\mathrm{m}^{2}$} \\
\hline Average $\pm \mathrm{SD}$ & $1.60 \pm 0.167$ & $1.70 \pm 0.174$ & 0.128 \\
\hline ECOG-PS & & & 1.000 \\
\hline $0 / 1 / 2 / 3 / 4$ & $9 / 3 / 0 / 0 / 0$ & $18 / 6 / 0 / 0 / 0$ & \\
\hline \multicolumn{4}{|l|}{ Laboratory data } \\
\hline White blood cell $\left(\times 10^{3} / \mathrm{mm}^{3}\right)$ & $5.73 \pm 1.04$ & $5.74 \pm 2.13$ & 0.728 \\
\hline Neutrophil $\left(\times 10^{3} / \mathrm{mm}^{3}\right)$ & $3.71 \pm 1.21$ & $3.64 \pm 1.74$ & 0.497 \\
\hline Hemoglobin (g/dL) & $11.2 \pm 2.50$ & $12.8 \pm 1.84$ & 0.026 \\
\hline Platelet $\left(\times 10^{4} / \mathrm{mm}^{3}\right)$ & $27.2 \pm 8.68$ & $29.1 \pm 12.4$ & 0.753 \\
\hline Total bilirubin (mg/dL) & $0.555 \pm 0.202$ & $0.546 \pm 0.277$ & 0.908 \\
\hline Serum creatinine $(\mathrm{mg} / \mathrm{dL})$ & $0.754 \pm 0.207$ & $0.798 \pm 0.196$ & 0.361 \\
\hline Albumin (g/dL) & $3.92 \pm 0.397$ & $4.23 \pm 0.493$ & 0.022 \\
\hline \multicolumn{4}{|l|}{ Site } \\
\hline Ascending colon & $4(33.3 \%)$ & $1(4.17 \%)$ & \\
\hline Transverse colon & $2(16.7 \%)$ & $0(0 \%)$ & \\
\hline Descending colon & $0(0 \%)$ & $3(12.5 \%)$ & \\
\hline Sigmoid colon & $3(25.0 \%)$ & $6(25.0 \%)$ & \\
\hline Rectum & $1(8.33 \%)$ & $10(41.7 \%)$ & \\
\hline Cecum & $2(16.7 \%)$ & $2(8.33 \%)$ & \\
\hline Appendix vermiformis & $0(0 \%)$ & $2(8.33 \%)$ & \\
\hline \multicolumn{4}{|l|}{ Stage } \\
\hline IIIa & $2(16.7 \%)$ & $5(20.8 \%)$ & \\
\hline $\mathrm{IIIb}$ & $6(50.0 \%)$ & $9(37.5 \%)$ & \\
\hline IIIc & $1(8.33 \%)$ & $4(16.7 \%)$ & \\
\hline IV (liver metastasis) & $3(25.0 \%)$ & $4(16.7 \%)$ & \\
\hline unclear & $0(0 \%)$ & $2(8.33 \%)$ & \\
\hline
\end{tabular}

SD: standard deviation, ECOG-PS: Eastern Cooperative Oncology Group Performance Status.

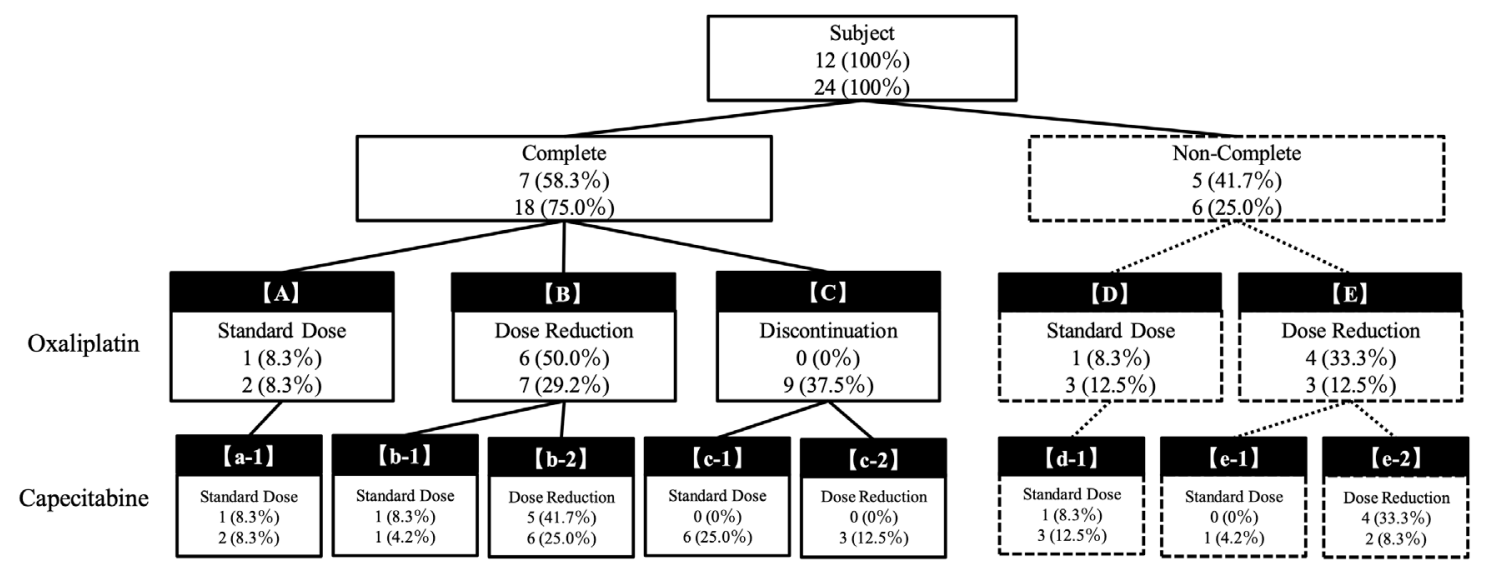

Figure 1. Course of treatment. Upper row; Elderly, Lower row; Non-Elderly. (A): Oxaliplatin Standard Dose, Complete. (B):Oxaliplatin Dose Reduction, Complete. (C):Oxaliplatin Discontinuation, Complete. (D):Oxaliplatin Standard Dose, NonComplete. (E):Oxaliplatin Dose Reduction, Non-Complete. (a-1):(A) and Capecitabine Standard Dose, Complete. (b-1):(B) and Capecitabine Standard Dose, Complete. (b-2):(B) and Capecitabine Dose Reduction, Complete. (c-1):(C) and Capecitabine Standard Dose, Complete. (c-2):(C) and Capecitabine Dose Reduction, Complete. (d-1):(D) and Capecitabine Standard Dose, Non-Complete. (e-1):(E) and Capecitabine Standard Dose, Non-Complete. (e-2):(E) and Capecitabin Dose Reduction, Non-Complete.

RDIs between the two groups for either oxaliplatin or capecitabine in patients who completed the 8 courses. These results suggest that it is possible to complete treatment while maintaining the therapeutic intensity even in patients over the age of 70 . On the other hand, discontinuation of treatment due to concomitant other diseases or progression of other diseases was more frequent in the elderly group, suggesting that it is necessary to intervene with elderly patients, paying attention not only to the occurrence of adverse 
events but also to the complications that may require discontinuation of treatment.

Anemia, hypoalbuminemia, hypomagnesemia, and habit of alcohol consumption are known to be

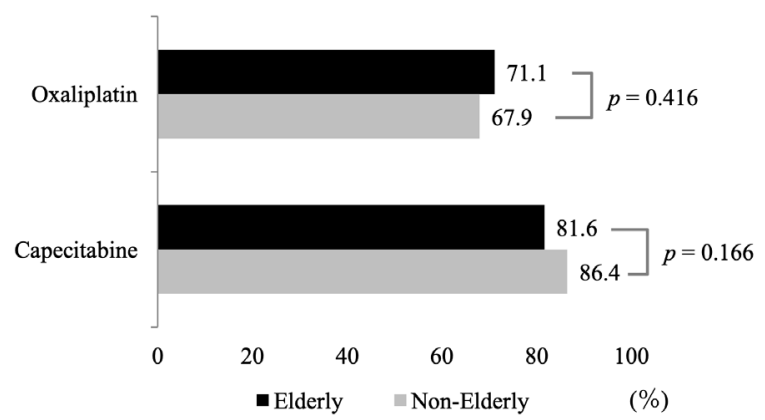

Figure 2. Median relative dose intensities (RDIs) in patients completed 8-courses. The median RDIs of oxaliplatin were $71.1 \%$ in the elderly and $67.9 \%$ in the non-elderly $(p=0.416)$. The RDIs of capecitabine were $81.6 \%$ and $86.4 \%$, respectively $(p=0.166)$. risk factors for peripheral neuropathy, especially in oxaliplatin-treated patients (10). In the present study, hemoglobin and albumin levels prior to the start of treatment were significantly lower in the elderly group. In addition, serum magnesium levels were often not included in the laboratory data prior to the start of treatment, and there were cases in which there were no medical records regarding alcohol consumption. Therefore, characteristics of the patients in this study were insufficient to confirm the risk factors for peripheral neuropathy. The hemoglobin and albumin levels suggest that the risk of peripheral neuropathy is high in the elderly group. However, the effects of peripheral neuropathy were lower in the elderly group than in the non-elderly group in the results of this survey; thus, we investigated the effects of age on peripheral neuropathy.

There have been several reports of the age-specific risks of oxaliplatin-induced peripheral neuropathy. A study comparing patients with colorectal cancer

Table 2. Reasons for dose reduction and discontinuation of oxaliplatin

\begin{tabular}{|c|c|c|c|c|c|c|c|c|c|c|c|c|c|c|c|c|c|}
\hline \multirow{4}{*}{ Reason } & \multicolumn{9}{|c|}{ Dose Reduction } & \multicolumn{8}{|c|}{ Discontinuation } \\
\hline & \multicolumn{4}{|c|}{ Elderly $(n=12)$} & \multicolumn{4}{|c|}{ Non-Elderly $(n=24)$} & \multirow[t]{3}{*}{$P$ value } & \multicolumn{4}{|c|}{ Elderly $(n=12)$} & \multicolumn{4}{|c|}{ Non-Elderly $(n=24)$} \\
\hline & \multicolumn{2}{|c|}{ All Grade } & \multicolumn{2}{|c|}{ Grade $\geq 3$} & \multicolumn{2}{|c|}{ All Grade } & \multicolumn{2}{|c|}{ Grade $\geq 3$} & & \multicolumn{2}{|c|}{ All Grade } & \multicolumn{2}{|c|}{ Grade $\geq 3$} & \multicolumn{2}{|c|}{ All Grade } & \multicolumn{2}{|c|}{ Grade $\geq 3$} \\
\hline & No. & $(\%)$ & No. & $(\%)$ & No. & $(\%)$ & No. & $(\%)$ & & No. & $(\%)$ & No. & $(\%)$ & No. & $(\%)$ & No. & $(\%)$ \\
\hline Hematologic toxicity & 6 & $(50.0)$ & 2 & $(16.7)$ & 6 & $(25.0)$ & 3 & $(12.5)$ & 0.157 & 0 & (0) & 0 & (0) & 2 & (8.3) & 1 & $(4.2)$ \\
\hline Neutropenia & 3 & $(25.0)$ & 0 & (0) & 5 & $(20.8)$ & 3 & $(12.5)$ & 1.000 & 0 & $(0)$ & 0 & (0) & 1 & $(4.2)$ & 1 & $(4.2)$ \\
\hline Thrombocytopenia & 5 & $(41.7)$ & 2 & $(16.7)$ & 3 & $(12.5)$ & 0 & (0) & 0.086 & 0 & (0) & 0 & (0) & 1 & $(4.2)$ & 0 & (0) \\
\hline Non-Hematologic toxicity & 4 & $(33.3)$ & 2 & $(16.7)$ & 14 & $(58.3)$ & 5 & $(20.8)$ & 0.157 & 0 & (0) & 0 & (0) & 7 & $(29.2)$ & 2 & (8.3) \\
\hline Peripheral neuropathy & 1 & $(8.3)$ & 0 & (0) & 9 & $(37.5)$ & 2 & $(8.3)$ & 0.115 & 0 & (0) & 0 & (0) & 7 & $(29.2)$ & 2 & (8.3) \\
\hline Anorexia & 2 & $(16.7)$ & 1 & $(8.3)$ & 1 & $(4.2)$ & 0 & (0) & 0.253 & 0 & (0) & 0 & (0) & 0 & (0) & 0 & (0) \\
\hline Diarrhea & 1 & $(8.3)$ & 1 & $(8.3)$ & 4 & $(16.7)$ & 2 & $(8.3)$ & 0.646 & 0 & (0) & 0 & (0) & 0 & (0) & 0 & (0) \\
\hline Nausea & 0 & (0) & 0 & (0) & 2 & (8.3) & 0 & (0) & 0.543 & 0 & (0) & 0 & (0) & 0 & (0) & 0 & (0) \\
\hline Malaise & 0 & (0) & 0 & (0) & 1 & $(4.2)$ & 1 & $(4.2)$ & 1.000 & 0 & (0) & 0 & (0) & 0 & (0) & 0 & (0) \\
\hline Infection & 1 & (8.3) & 0 & (0) & 1 & $(4.2)$ & 0 & (0) & 1.000 & 0 & (0) & 0 & (0) & 0 & (0) & 0 & (0) \\
\hline
\end{tabular}

There is some overlapping. The severity of adverse events was assessed according to the Common Terminology Criteria for Adverse Events, version 5.0. The worst grade considered at least possibly related to treatment is given. The $P$ values are for the differences between the groups for all grades.

Table 3. Reasons for dose reduction of capecitabine

\begin{tabular}{|c|c|c|c|c|c|c|c|c|c|}
\hline \multirow{3}{*}{ Reason } & \multicolumn{4}{|c|}{ Elderly $(n=12)$} & \multicolumn{4}{|c|}{ Non-Elderly $(n=24)$} & \multirow[t]{3}{*}{$P$ value } \\
\hline & \multicolumn{2}{|c|}{ All Grade } & \multicolumn{2}{|c|}{ Grade $\geq 3$} & \multicolumn{2}{|c|}{ All Grade } & \multicolumn{2}{|c|}{ Grade $\geq 3$} & \\
\hline & No. & $(\%)$ & No. & $(\%)$ & No. & $(\%)$ & No. & $(\%)$ & \\
\hline Hematologic toxicity & 4 & $(33.3)$ & 2 & $(16.7)$ & 5 & $(20.8)$ & 3 & (12.5) & 0.443 \\
\hline Neutropenia & 1 & $(8.3)$ & 0 & (0) & 4 & $(16.7)$ & 3 & $(12.5)$ & 0.646 \\
\hline Thrombocytopenia & 3 & $(25.0)$ & 2 & $(16.7)$ & 2 & $(8.3)$ & 0 & (0) & 0.307 \\
\hline Non-Hematologic toxicity & 6 & $(50.0)$ & 2 & $(16.7)$ & 7 & $(29.2)$ & 3 & $(12.5)$ & 0.281 \\
\hline Anorexia & 1 & $(8.3)$ & 0 & (0) & 1 & $(4.2)$ & 0 & (0) & 1.000 \\
\hline Diarrhea & 1 & (8.3) & 1 & (8.3) & 4 & $(16.7)$ & 2 & $(8.3)$ & 0.646 \\
\hline Hand-foot syndrome & 3 & $(25.0)$ & 1 & (8.3) & 2 & $(8.3)$ & 1 & $(4.2)$ & 0.307 \\
\hline Infection & 1 & $(8.3)$ & 0 & (0) & 0 & (0) & 0 & (0) & 0.333 \\
\hline
\end{tabular}

There is some overlapping. The severity of adverse events was assessed according to the Common Terminology Criteria for Adverse Events, version 5.0. The worst grade considered at least possibly related to treatment is given. The $P$ values are for the differences between the groups for all grades. 
aged 50 to 68 years with those over the age of 69 who were treated with adjuvant chemotherapy or firstline treatment for metastatic disease reported no ageassociated statistically significant difference in acute or chronic peripheral neuropathy (11). On the other hand, in a study of patients with colorectal cancer who were treated with adjuvant chemotherapy, the risk of peripheral neuropathy was reported to be higher in patients over the age of 70 than in those aged 66 to 69 years (12). It has also been reported that the duration of peripheral neuropathy was longer in patients under the age of 60 than in those over the age of 60 (10). Thus, there is no consensus on the risks of oxaliplatin-induced peripheral neuropathy with respect to age.

The effects of peripheral neuropathy were lower in the elderly group than in the non-elderly group in our study. The reason for this may be due to the effect of hematologic toxicity. In the elderly group, hematologic toxicity was the reason for $50.0 \%$ of all cases of oxaliplatin dose reductions. In the elderly, the decline in bone marrow function associated with aging may lead to more severe hematologic toxicity and prolonged recovery (13). In the present study, there was no significant difference between the elderly and non-elderly groups in the blood cell counts prior to the start of CAPOX therapy except for the hemoglobin level. This suggests that elderly patients may have been more susceptible to hematopoietic exhaustion due to chemotherapy, and may have experienced less peripheral neuropathy due to dose reduction resulting from hematologic toxicity in response to more severe hematologic toxicity and prolonged recovery before the reduction resulting from peripheral neuropathy.

The limitations of this study include the small number of cases; hence, further research is needed on a larger number of patients. Second, we were unable to evaluate efficacy in terms of overall survival or diseasefree survival, because there has been insufficient time since completing adjuvant CAPOX therapy. In addition, it was not possible to retrospectively investigate patient adherence with capecitabine, which is an oral anticancer drug. The RDI for capecitabine was calculated based on the number of prescription days, and the RDI may possibly be even lower in patients with poor adherence. Especially in the elderly, who are often treated by polypharmacy due to chronic diseases, in addition to a decline in activities of daily living and cognitive function (13), it is important to ensure adherence in order to maintain the therapeutic intensity of CAPOX therapy. In future, it will be necessary to consider including a survey of adherence.

In conclusion, the results of this study suggested that a similar therapeutic intensity can be maintained in elderly patients relative to non-elderly patients by appropriate dose reduction and discontinuation of drug treatments. Elderly patients are more susceptible to hematologic toxicity than to peripheral neuropathy.

\section{Funding: None.}

Conflict of Interest: Y.Y. has received honoraria from Chugai, Taiho, and Nipponkayaku, Japan. The other authors declare that they have no conflicts of interest.

\section{References}

1. National Cancer Center, Center for Cancer Control and Information Services. https://ganjoho.jp/reg_stat/statistics/ stat/summary.html (accessed December 25, 2020).

2. Haller DG, Tabernero J, Maroun J, de Braud F, Price T, Van Cutsem E, Hill M, Gilberg F, Rittweger K, Schmoll HJ. Capecitabine plus oxaliplatin compared with fluorouracil and folinic acid as adjuvant therapy for stage III colon cancer. J Clin Oncol. 2011; 29:1465-1471.

3. Yothers G, O'Connell MJ, Allegra CJ, Kuebler JP, Colangelo LH, Petrelli NJ, Wolmark N. Oxaliplatin as adjuvant therapy for colon cancer: updated results of NSABP C-07 trial, including survival and subset analyses. J Clin Oncol. 2011; 29:3768-3774.

4. Tournigand C, André T, Bonnetain F, Chibaudel B, Lledo G, Hickish T, Tabernero J, Boni C, Bachet JB, Teixeira L, de Gramont A. Adjuvant therapy with fluorouracil and oxaliplatin in stage II and elderly patients (between ages 70 and 75 years) with colon cancer: subgroup analyses of the Multicenter International Study of Oxaliplatin, Fluorouracil, and Leucovorin in the Adjuvant Treatment of Colon Cancer trial. J Clin Oncol. 2012; 30:3353-3360.

5. Haller DG, O'Connell MJ, Cartwright TH, Twelves CJ, McKenna EF, Sun W, Saif MW, Lee S, Yothers G, Schmoll HJ. Impact of age and medical comorbidity on adjuvant treatment outcomes for stage III colon cancer: a pooled analysis of individual patient data from four randomized, controlled trials. Ann Oncol. 2015; 26:715724.

6. Nitsche U, Stöss C, Friess H. Effect of adjuvant chemotherapy on elderly colorectal cancer patients: lack of evidence. Gastrointest Tumors. 2017; 4:11-19.

7. Grothey A, Sobrero AF, Shields AF, et al. Duration of adjuvant chemotherapy for stage III colon cancer. N Engl J Med. 2018; 378:1177-1188.

8. Yoshino T, Yamanaka T, Oki E, et al. Efficacy and longterm peripheral sensory neuropathy of $3 v s 6$ months of oxaliplatin-based adjuvant chemotherapy for colon cancer: the ACHIEVE phase 3 randomized clinical trial. JAMA Oncol. 2019; 5:1574-1581.

9. Japanese Society of Medical Oncology, Japan Society of Clinical Oncology. Clinical Practice Guidelines of Cancer Drug Therapies for the Elderly. Nankodo, Tokyo, Japan, 2019; pp.25-28.

10. Vincenzi B, Frezza AM, Schiavon G, Spoto C, Silvestris N, Addeo R, Catalano V, Graziano F, Santini D, Tonini G. Identification of clinical predictive factors of oxaliplatininduced chronic peripheral neuropathy in colorectal cancer patients treated with adjuvant Folfox IV. Support Care Cancer. 2013; 21:1313-1319.

11. Argyriou AA, Briani C, Cavaletti G, Bruna J, Alberti P, Velasco R, Lonardi S, Cortinovis D, Cazzaniga M, Campagnolo M, Santos C, Kalofonos HP. Advanced age and liability to oxaliplatin-induced peripheral neuropathy: post hoc analysis of a prospective study. Eur J Neurol. 2013; 20:788-794.

12. Raphael MJ, Fischer HD, Fung K, Austin PC, Anderson 
GM, Booth CM, Singh S. Neurotoxicity outcomes in a population-based cohort of elderly patients treated with adjuvant oxaliplatin for colorectal cancer. Clin Colorectal Cancer. 2017; 16:397-404.e1.

13. Hamaguchi T. Risk assessment of chemotherapy for the elderly patients with gastrointestinal cancer. Gan To Kagaku Ryoho. 2018; 45:12-15. (in Japanese)

Received February 12, 2021; Revised December 3, 2021;
Accepted December 22, 2021.

Released online in J-STAGE as advance publication Janiary 17, 2022.

*Address correspondence to:

Ayumi Tsuchiya, Pharmaceutical Department, Center Hospital of the National Center for Global Health and Medicine, 1-21-1 Toyama, Shinjuku-ku, Tokyo 162-8655, Japan.

E-mail: atsuchiya@hosp.ncgm.go.jp 